\title{
'You just prefer to die early!': how socioecological context impedes treatment for people living with HIV in Iran
}

\author{
Vira Ameli, ${ }^{1,2}$ Leila Taj, ${ }^{2}$ Jane Barlow, ${ }^{1}$ Lora Sabin, ${ }^{3}$ Franziska Meinck,,${ }^{4,5}$ \\ Jessica Haberer, ${ }^{6}$ Minoo Mohraz ${ }^{2}$
}

\begin{abstract}
To cite: Ameli V, Taj L, Barlow J, et al. 'You just prefer to die early!': how socioecological context impedes treatment for people living with HIV in Iran. BMJ Global Health 2021;6:e006088. doi:10.1136/ bmjgh-2021-006088
\end{abstract}

Handling editor Stephanie M Topp

Received 29 April 2021 Accepted 25 October 2021

Check for updates

(c) Author(s) (or their employer(s)) 2021. Re-use permitted under CC BY-NC. No commercial re-use. See rights and permissions. Published by BMJ.

${ }^{1}$ Social Policy and Intervention, Oxford University, Oxford, UK ${ }^{2}$ Iranian Research Center for HIV / AIDS, Tehran University of Medical Sciences, Tehran, Iran (the Islamic Republic of) ${ }^{3}$ Department of Global Health, Boston University School of Public Health, Boston, Massachusetts, USA

${ }^{4}$ School of Social and Political Science, University of Edinburgh Edinburgh, UK

${ }^{5}$ School of Public Health, NorthWest University, Potchefstroom, South Africa

${ }^{6}$ Center for Global Health at Massachusetts General Hospital, Massachusetts General Hospital, Boston, Massachusetts, USA

Correspondence to

Vira Ameli;

vira.ameli@spi.ox.ac.uk

\section{ABSTRACT}

Introduction Despite the low prevalence of HIV and broad provision of antiretroviral therapy, the Middle East and North Africa (MENA) remains the only region where new HIV infections and AIDS-related deaths are not declining. There is a dearth of evidence from MENA on antiretroviral therapy engagement. In this qualitative study, we sought to identify the ways in which successful treatment is hindered in Iran, which is home to $24 \%$ of HIV infections in MENA.

Methods From August 2018 to January 2019, we used purposive sampling and conducted 12 individual interviews and 8 focus group discussions with 27 female and 31 male patients, in addition to 5 individual interviews with HIV care providers and 1 focus group discussion with 8 care providers. Social constructivism augmented with realistinformed thematic analysis was used to understand how the socioecological context triggers cognitive and affective mechanisms that disrupt antiretroviral therapy.

Results The use of Thematic Network Analysis resulted in the identification of three key cognitive and affective mechanisms that appear to shape treatment experience and are triggered via HIV's socioecological context and changing economic conditions in Iran: denial in response to societal negative perceptions of HIV; fear in response to societal lack of awareness regarding HIV and misinformation; and despair in response to HIV-related stigma and enacted discrimination, economic insecurity and social support.

Conclusions To our knowledge, this is the first study within MENA to identify pathways through which successful treatment is hindered. It appears that lack of societal awareness regarding HIV is specific to low prevalence settings, such as MENA countries, where negative perceptions, stigma, discrimination and misinformation regarding HIV and its treatment produce denial, fear and despair, acting as mechanisms that disrupt antiretroviral therapy. The experience of despair, in response to changing economic conditions and social support, further impacts treatment experience.

\section{INTRODUCTION}

The Middle East and North Africa (MENA) region is considered to be a 'black-hole' in terms of HIV/AIDS research. ${ }^{1}$ Despite provision of antiretroviral therapy, new HIV

\section{WHAT IS ALREADY KNOWN?}

$\Rightarrow$ Globally, adherence to antiretroviral therapy is impacted by political, sociostructural, institutional, interpersonal and individual factors.

$\Rightarrow$ Within the Middle East and North Africa region, a knowledge gap exists in terms of the context of engagement with and adherence to antiretroviral therapy.

\section{WHAT ARE THE NEW FINDINGS?}

$\Rightarrow$ Three key cognitive and affective mechanisms of denial, fear and despair were found to interfere with successful antiretroviral therapy among Iranians living with HIV.

$\Rightarrow$ Socioecological pathways that trigger denial, fear and despair are detailed and considered within the changing economic conditions of sanctions and inflation.

\section{WHAT DO THE NEW FINDINGS IMPLY?}

$\Rightarrow$ Low prevalence of HIV stifles public knowledge about HIV and heightens stigma, discrimination and negative perceptions that afflict people living with HIV.

$\Rightarrow$ Reducing or intentionally stopping adherence to antiretroviral therapy appears to be manifested in climates of economic insecurity that increase despair and suicidal thoughts.

infections continue to rise and AIDS-related deaths remain stagnantly high in most MENA countries, in contrast to the rest of the world. ${ }^{2}$ Only $67 \%$ of people diagnosed with HIV in MENA receive treatment, and of those only $83 \%$ are reported to be virally suppressed, indicating that engagement with care and adherence to treatment are key contributing factors to HIV-related outcomes.

Yet, there is a dearth of evidence from MENA countries on the lived experience of engaging with lifelong treatment. ${ }^{3-6}$ Of the few studies conducted within MENA, 
most focus on prevalence or biomedical aspects of the condition, ${ }^{7-10}$ and studies of adherence focus on identifying key affected populations, such as people who use drugs and imprisoned individuals at risk of nonadherence. ${ }^{11} 12$ Quantitative and biomedical studies promote understanding about the prevalence and risk profiles of non-adherence among key affected groups, ${ }^{1314}$ and can increase knowledge on drug resistance profiles to antiretroviral therapy in different settings. ${ }^{8}$ Yet, there is a need for qualitative studies for identifying how and why engagement with and adherence to treatment decline, in addition to elucidating the pathways that lead from the sociocultural, political and economic context to individual reasonings and reactions that determine behaviour.

Studies from other regions provide evidence within the socioecological framework of multiple influences on lifelong antiretroviral use. ${ }^{15-20}$ First, at a political level, health policies, incriminating laws and public health priorities influence HIV risk behaviours. ${ }^{21}$ Second, at a sociostructural level, poverty, ${ }^{22}$ HIV stigma, ${ }^{23}{ }^{24}$ violence victimisation $^{25}$ and cultural norms such as masculine stoicism ${ }^{26}$ can act as barriers to consistent antiretroviral therapy. Third, at an institutional level, appropriate services, quality of care, competent staff, supportive health workers and sufficient resources contribute to engagement in care and adherence to treatment. ${ }^{27}{ }^{28}$ Fourth, at an interpersonal level, social support, ${ }^{29}$ relationship intimacy and personal satisfaction can impact adherence behaviour. ${ }^{30}$ Fifth, the aforementioned contextual levels, directly and indirectly, shape the situations in which individuals cope, experience emotions, use drugs and engage with lifelong adherence. ${ }^{31-34}$ Moreover, factors within a socioecological framework must be considered within the temporal junctures in which they meet; changes in political climate and policy directions could be a key contributor in shaping the coalescence of the influencing factors within individual lives.

This study was conducted using a social constructivist approach, augmented with a realist-informed analysis, to thematically investigate the pathways from the socioecological context to individual cognitive and affective mechanisms that impede treatment for people living with HIV in Tehran, Iran. The research question for this study was how individuals react to their context in ways that shape their adherence behaviours. The qualitative nature of the study allowed consideration of the impact of the time period in which the study took place, including, for example, the increasing economic sanctions and inflation that exacerbated financial and psychological hardships. Augmenting the constructivist paradigm with a realist informed analysis enhanced the transferability of context-specific findings. Iran has $24 \%$ of HIV cases within the MENA region, ${ }^{2}$ and provides universal access to antiretroviral therapy, constituting a useful case study for exploring the socioecological pathways that shape engagement with antiretroviral therapy.

\section{HIV epidemic in Iran}

The HIV epidemic in Iran was initially driven through shared needles, but is now primarily driven through sexual transmission. ${ }^{35}$ It is estimated that 54 thousand people are living with HIV in Iran, a country with a total population of 85 million. By 2020, $42 \%$ of those living with HIV knew their status, of which $68 \%$ were receiving treatment. Only $81 \%$ of antiretroviral recipients achieved viral suppression in 2018, the only year in which this target was reported. ${ }^{2}$ Iran provides universal and free access to treatment through a decentralised model of health delivery across 31 provinces via university hospitals, operating under the joint Ministry of Health and Medical Education, providing uniform services for HIV, including voluntary counselling and testing (VCT) centres and positive clubs for psychosocial support.

Failure to meet the 90-90-90 targets by 2020 and the loss of people along the care cascade, despite free provision of test-and-treat, signify socioecological barriers to diagnosis and care. Fear of prosecution and stigmatisation deter many from seeking HIV testing and treatment, despite immunity offered by patient confidentiality. Men who have sex with men are prosecuted only when there is a petition from a victim of sexual offence, and independent investigation of homosexual sex by police or prosecutors is subject to punishment by law. Nevertheless, perceived threat of prosecution and sentencing continues to deter many from reaching services. For additional information, see online supplemental appendix 1 .

\section{METHODS \\ Design and setting}

Qualitative methods are useful for situating behaviour within the wider social context and generating roadmaps for intervention design. ${ }^{36-39}$ This social constructivist qualitative study was augmented by realist-informed analytical thinking for a deeper understanding of how behaviours are produced via context-specific cognitive and affective mechanisms that cannot be measured quantitatively. ${ }^{40}$ Merging two research paradigms: social constructivism with realism, allowed the identification of cognitive and affective mechanisms as reactions to socioecological triggers that may be socially constructed, but remain rooted in a shared reality, with transferable lessons to similar contexts. ${ }^{41} 42$ Rather than seeking to generate standard characterisations of all members of a statistically representative sample, a social constructivist approach was used to answer the research question through an in-depth exploration of the diverse ways that antiretroviral users in this setting were responding to the socioecological context of living with HIV in Iran. A realist-informed thematic analysis was then applied to produce identifiable mechanisms that appear to shape HIV treatment adherence and engagement within Iranian socioecological context. Thus, we were able to study how the understandings and actions of people living with HIV shape their engagement with and adherence to treatment, by 
exploring their collectively constructed representational realities, and their reactions to it, instead of surveying a list of individual and societal characteristics as decontextualised properties of a closed social system. The identified mechanisms and context-specific findings of this study will inform a future mixed-methods trial and realist evaluation of an intervention to be empirically tested within this population.

The study is part of an ongoing research project to understand and improve antiretroviral use of Iranians living with HIV, conducted at the infectious diseases unit of the Imam Khomeini Hospital Complex (IKHC), which is the teaching hospital of Tehran University of Medical Sciences and based in the sociodemographically diverse city centre of Tehran. IKHC houses Tehran's central VCT centre, where participants of this study were recruited from over 4000 patients. Additional psychosocial support is offered on-site at the peer-run positive club, a supportive location in which the study interviews took place. IKHC also houses the Iranian Research Centre for HIV/AIDS (IRCHA), the main collaborating research partner of this project, with a team of eight fulltime researchers and research assistants, from which the staff for this project were recruited. Recruitment of study participants took place from August 2018 to January 2019. All staff members conducting the recruitment and informed consent process were trained in the ethical treatment of human participants and were familiar with informed consent guidelines. Recruitment was indirect by asking healthcare providers to provide a brief introduction to the study to patients during individual clinic visits. Patients who showed interest were then invited to visit IRCHA to provide written and informed consent, with the agreement that their identities would not be revealed. A meal was provided to show appreciation of the participant's contribution to the study, on the day of the interview.

The qualitative study draws on the perspectives of both recently diagnosed and more experienced active antiretroviral users, and their healthcare providers. The research method involved interviews and focus group discussions (FGDs), both of which allow researchers to explore areas of interest, although with important differences in terms of approach. For instance, individual interviews allow an in-depth exploration of personal and sensitive topics, while FGDs involve group interactions with the possibility to assess consensus and dissent within a trusted environment among peers. ${ }^{43}$ The FGDs were conducted separately for men and women to explore any differences between genders in their reactions to contextual triggers and the impact on antiretroviral therapy engagement. FGDs and interviews were recorded with the consent of participants, who chose their own nicknames to be used during the recording.Following transcription, the audio recorded files of participants' voices were deleted and no information with personal identifiers were stored or accessible.

\section{Sampling and analysis}

Purposive sampling ${ }^{44}$ was used to recruit participants with a range of experience in terms of adherence to antiretroviral therapy, based on information in clinic records of people living with HIV receiving antiretroviral therapy, using the following criteria: (1) antiretroviral recipients diagnosed within the past year, (2) antiretroviral recipients diagnosed more than 1 year ago; (3) antiretroviral recipients who experienced disruptions in care and adherence and (4) antiretroviral recipients who had to change more than one treatment regimen due to drug resistance. For each of these criteria, men and women were sampled separately from clinical records. Participants were phone-called by research staff, who were trained in informed consent process and introduced to the research project. An overview of information included in the consent forms was provided with regard to the study purpose and design and details regarding the length, location and general discussion points for the qualitative interviews. If interested, three dates were provided to choose for participation. A reminder phonecall was made 1 day prior to the scheduled date. On the day of the interview, participants were given informed consent sheets to read carefully before signing.

Twelve individual interviews and 8 FGDs with a total of 27 female and 31 male patients were conducted. In addition, five individual interviews, with HIV care providers (two physicians, one counsellor, two peer supporters who were living with HIV) and one FGD, with a total of eight additional care providers (six physicians, one counsellor, one nurse), were conducted. Focus groups had an average of six participants, with the aim of creating a smaller and more intimate environment for conversations. Individual interviews averaged $40 \mathrm{~min}$ and focus groups averaged 1.5 hours. Table 1 presents a summary of the characteristics of these participants.

Semistructured topic guides focused on what the socioecological barriers and facilitators to antiretroviral therapy adherence are-and how and why they impact antiretroviral adherence and use (Topic guides also covered potential approaches for intervention and current clinic support mechanisms; however, these are not discussed in detail in this paper.). Interviews were conducted by the lead researcher (VA), a native Persianspeaker and audiorecorded with permission from the participants. These recordings were subsequently transcribed in Persian by VA and imported into MAXQDA, a qualitative analysis software package, for organising and coding the text segments with descriptive headings. Two researchers (VA and LT) coded the data independently in Persian. The emerging codes were reviewed in intervals, after coding twenty per cent of the transcripts, by discussing differences in coding to reach an agreement. Differences focused largely on different meanings being attributed to specific words rather than disagreements about the emerging themes, thereby requiring alignment of language more than the need to resolve disagreements 
Table 1 Participant characteristics

\begin{tabular}{|c|c|c|c|c|}
\hline Study characteristics & Antiretroviral recipients & Healthcare providers & Peer supporters & Total \\
\hline \multicolumn{5}{|l|}{ Research participation type } \\
\hline Individual interview, no. & 12 & 3 & 2 & 17 \\
\hline Women, no. & 4 & & & \\
\hline Men, no. & 8 & & & \\
\hline Focus group, total no. (no. of groups) & $58(8)$ & $8(1)$ & 0 & 58 \\
\hline Women, no. (no. of groups) & $27(4)$ & & & \\
\hline Men, no. (no. of groups) & $31(4)$ & & & \\
\hline Total participants, no. & 70 & 11 & 2 & 75 \\
\hline Mean age, years & 38 & 58 & 41 & \\
\hline Women, no. (\%) & $31(44)$ & & & \\
\hline Men, no. (\%) & $39(55)$ & & & \\
\hline
\end{tabular}

about what the data meant. The agreed on themes were then translated into English.

Thematic network analysis was employed to summarise the main themes within web-like networks that illustrated pathways from the specific socioecological context of living with HIV in Iran to individual cognitive and affective mechanisms that impact treatment uptake and adherence. Following the prescribed steps for thematic network analysis ${ }^{45}$ the transcribed texts were first coded with a basic code, informed by the socioecological framework of multilevel factors shaping antiretroviral therapy success; the basic codes were then clustered into higher order basic themes, identifying how the socioecological context produced specific cognitive and affective mechanisms of treatment uptake and adherence; the basic themes were then grouped by the identified contextual triggers into organising themes, which were then grouped by the cognitive and affective mechanisms into global themes. The outcome of this thematic network analysis is presented in figure 1, highlighting

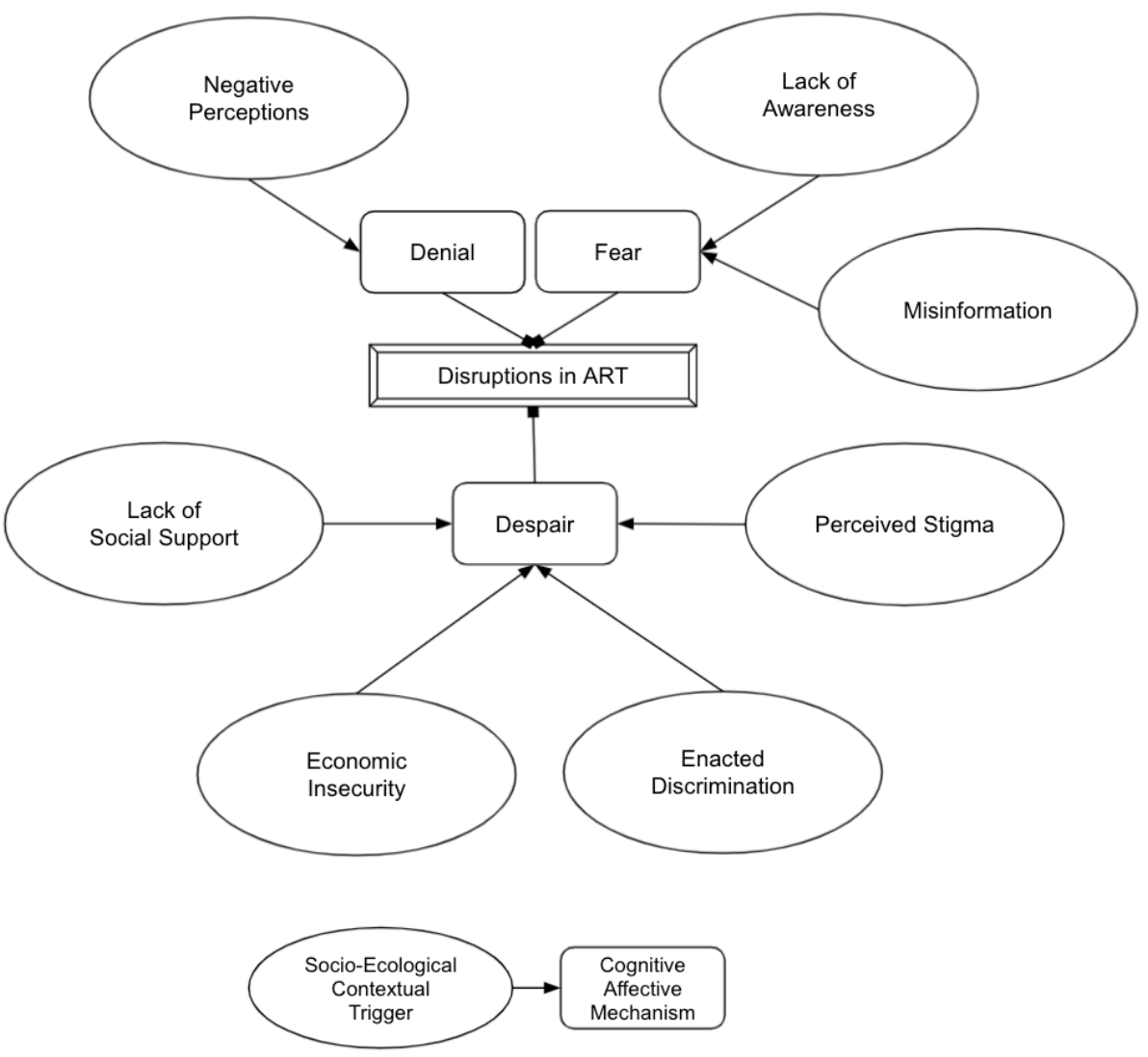

Figure 1 Pathways from socioecological contextual triggers (organising themes) to cognitive and affective mechanisms (global themes) that disrupt antiretroviral therapy (ART). 
Table 2 Thematic network analysis of basic, organising and global themes

\begin{tabular}{|c|c|c|}
\hline $\begin{array}{l}\text { Global } \\
\text { themes } \\
\text { Mechanisms }\end{array}$ & $\begin{array}{l}\text { Organising themes } \\
\text { Contextual triggers }\end{array}$ & $\begin{array}{l}\text { Basic themes } \\
\text { How context triggers mechanisms }\end{array}$ \\
\hline $\begin{array}{l}\text { Denial } \\
\text { cognitive }\end{array}$ & Negative perceptions & $\begin{array}{l}\text { Denial can be a coping mechanism to adjust to negative social perception of HIV } \\
\text { Staying in denial can delay treatment and encourage harmful drug use. }\end{array}$ \\
\hline \multirow{2}{*}{$\begin{array}{l}\text { Fear } \\
\text { affective }\end{array}$} & Lack of awareness & Fear appears to be caused by lack of societal awareness of HIV and ART. \\
\hline & Misinformation & Fear can be triggered as a result of misinformation. \\
\hline \multirow[t]{4}{*}{$\begin{array}{l}\text { Despair } \\
\text { affective }\end{array}$} & Perceived stigma & $\begin{array}{l}\text { Despair appears to be felt in response to perceived stigma against HIV status. } \\
\text { Newly diagnosed patients can experience stronger despair, which can cause } \\
\text { depression. }\end{array}$ \\
\hline & Enacted discrimination & $\begin{array}{l}\text { Despair can be an emotional response to acts of discrimination by the society. } \\
\text { Discrimination by families and medical society can produce stronger despair. }\end{array}$ \\
\hline & Economic insecurity & Despair can be an emotional response to economic insecurity. \\
\hline & Social support & $\begin{array}{l}\text { Despair appears to be felt when social support is lacking. } \\
\text { Supportive family and services can moderate feelings of despair. }\end{array}$ \\
\hline
\end{tabular}

the pathways that produce context-specific cognitive and affective mechanisms, via the main themes that address the research question and build the structure for the presentation of our findings.

\section{RESULTS}

The findings of our study identify cognitive and affective mechanisms that hinder adherence to antiretroviral therapy situated within the socioecological context of living with HIV in Iran, as opposed to decontextualised individual reasonings and reactions. The mechanisms specific to, and triggered within, the socioecological context of living with HIV in Iran appear to be: denial in response to societal negative perceptions of HIV; fear in response to societal lack of awareness regarding HIV and misinformation, and despair in response to HIVrelated stigma and enacted discrimination, economic insecurity and lack of social support. Thematic pathways from socioecological contextual triggers to cognitive and affective mechanisms that disrupt antiretroviral therapy are depicted diagrammatically in figure 1 and summarised in table 2 . The results section is presented in terms of the cognitive and affective mechanisms, within which are located further subsections based on socioecological contextual triggers. The mechanisms are presented in the order that they appear to be experienced as navigating through HIV care cascade after diagnosis: first experiencing denial and fear, followed by despair. For each cognitive and affective mechanism, a summary of gender differences is provided, as assessed by the extent to which each theme was emphasised among men versus women.

\section{Denial as a cognitive mechanism}

Denial was identified as a cognitive mechanism underpinning the rejection of the HIV diagnosis and need for treatment, motivated by the need to escape the negative societal perceptions regarding HIV. This mechanism was triggered in the initial stages of the HIV care cascade, exposing HIV diagnosed patients to an increased risk of harmful drug use due to the need to escape from the new reality of living with HIV. Denial appears to be the primary mechanism producing a lengthy gap between diagnosis and enrolment in care. As a coping mechanism, male interviewees commonly reported referring to denial and were more likely to use words such as 'not being in acceptance' and 'being in denial'.

\section{Negative societal perceptions regarding HIV}

A number of interviewees described the ways in which negative perceptions regarding HIV in the wider society triggered a state of denial following diagnosis. To avoid any association with the condition, some participants chose to not initiate or discontinue treatment.

The perception that people give is very negative. It's like it feels better to stay in denial even if you have it, to run away, but you don't realise that you are hurting yourself more. (Male patient-focus group 5)

The name of this disease is more negative than anything in our society. Because of it they blame you and embarrass you. It's not like cancer or anything else that you are the victim. (Male patient-interview 1 )

Denial appeared to have hindered the decision to enrol in care in the first place, delaying the initiation of care until the point that CD4 levels were too low.

It took me exactly six years to come out of the denial stage. When I started treatment my CD4 was 8, I couldn't walk, I couldn't breathe. I couldn't move. It's like you are in that denial stage in the beginning. Until you accept HIV, make other friends [who live with HIV], get treatment and get help. (Male patient-interview 7)

Using drugs, particularly trying new drugs and new routes of administration, were also described as a form 
of escape from the new reality of living with HIV, with significant long-term consequences for those who used more lethal types of drugs.

I had a friend who passed away. He went towards these new substances [methamphetamines] that are completely different from the traditional substances [opiates]. In onemonth, they [methamphetamines] do the harm of 10 years of substance use. Before he exercised, he was in shape. But after his HIV diagnosis he just drowned himself in these substances [methamphetamine] and was soon gone. (Male patient-focus group 1)

\section{Fear as an affective mechanism}

Fear was identified as an affective mechanism in response to HIV treatment being perceived as daunting, and thereby avoided. As incorrect information is propagated through society, fear can spread easily among people living with HIV. More women, than men, cited fear as a mechanism of avoiding treatment.

\section{Lack of societal awareness regarding HIV}

Participants talked about a general lack of awareness of HIV in Iranian society due in part to the fact that HIVrelated topics are not widely discussed in the society, thereby many people have never heard about HIV, still think of it as a death sentence, and lack knowledge with regards to the availability of treatment. The interviews indicated that existing knowledge about HIV tended to be superficial, and that the condition itself was perceived to be stigmatising, leading to fear and a disinclination to seek treatment.

When I found out I have HIV I was shocked and afraid. I never even thought that in Iran anyone has HIV. I thought it only happens in other countries or to people who act transgressively. (Female patient-interview 4)

People are not even aware that now you can get treated and view [HIV] with fear. They don't see a difference between HIV and AIDS. You will be labelled [pejoratively] as 'AIDS-y'. This makes it scary to show up appointments. You just prefer to stay away. (Male patient-interview 10)

\section{Misinformation}

Participants indicated that a range of issues related to medications could trigger fear and mistrust of antiretroviral therapy. Some described fear of long-term therapy affecting their ability to adapt to daily medication schedules.

I really don't like medicines in general, so the thought that I have to take 60 pills every month was scary to me and it took me a long time to get used to this. (Female patientfocus group 8)

I was really scared of these drugs [antiretrovirals]. I wasn't sure how sure I could be about how safe and useful they are. (Female-interview 5)

A peer counsellor recounted misinformation, false beliefs, and conspiracy theories, being disseminated among patients through waiting rooms and online chatrooms.

Some patients don't even believe in chemical medications. From the beginning, they go towards traditional medicine and say why should we take these drugs. They think they've been 'turned into lab mice'. This is their perspective. They also find scary information online and share with others in waiting rooms and chat groups. (Female peer counsellorinterview 1).

Concerns regarding treatment side effects appeared often to be shared leading to misinformation and triggering fear of treatment initiation in new patients.

When they speak about the side-effects of medications, they create such fear that new patients sometimes delay beginning treatment, or they won't start until they have become very ill. Sometimes patients start to imagine they are experiencing side effects, for example saying that 'the drugs do not descend from their throat into their stomach'. (Female peer counsellor-interview 1).

\section{Despair as an affective mechanism}

Despair was identified as an affective mechanism that led to treatment adherence being perceived as meaningless and, subsequently, deprioritised. The societal context of HIV-related stigma, enacted discrimination against HIV status and economic insecurity appeared to be the key contextual triggers for despair, sometimes causing depression. However, social support appeared to mitigate some of the despair that resulted from contextual adversities, while lack of support engendered more despair. Despair, as an underpinning motivator of e with treatment was commonly referred to by female interviewees, especially in the context of family problems. Some women intentionally stopped adherence when they felt uncared for and depressed. Female respondents were more likely to use words such as 'wanting to give up on life' and 'preferring to die early'.

\section{HIV-related perceived stigma}

Participants described how from the onset of HIV diagnosis, the experience of HIV-related stigma and the feeling of being rejected by society, created a sense of despair and in some cases depression, weakening the motivation to continue adherence with treatment.

This adherence to treatment requires motivation, hope. But a big problem that we have is problems which stem from the stigma [of HIV]. Sometimes the pressures from stigma and the attitude of the society are so strong that you just lose hope and want to end the whole process of treatment. And it has happened to many of us. (Female patient-interview 2)

Indeed, the interview data suggest that stigma widely disrupts the social conditions in which people living with HIV interact with society and form relationships.

Stigma in this society is what makes everything in life more difficult for us, from medical treatment to the possibility of marriage. (Male patient-focus group 5) 
The first year following diagnosis appeared to be the most sensitive period, in which the shock of being diagnosed with HIV combined with the experience of stigma contributed to the erosion of hope. Some participants appeared to have internalised the stigma in the early days, leading to isolation and depression, further diminishing adherence.

Unfortunately, in our society we have to live with this stig$\mathrm{ma}$, and in the beginning, you also believe this stigma yourself, so you are more depressed and live a hopeless life. (Female patient-focus group 4)

The early days were terrible. You know about stigma, but you do not expect to feel so much emotional pain because of it. You just retreat within yourself and this impacts your ability to function, let alone taking your treatment seriously. (Female patient-focus group 2)

\section{Enacted discrimination against HIV status}

Acts of discrimination against HIV were described to be present in all segments of the society and identified as a key trigger for despair.

When people discriminate against us [patients living with HIV] it's like the higher the education levels, the lower the 'sho'oor' (social intelligence), so you really lose hope. (Female-focus group 4)

My only problem is feeling depressed because people in the society do not accept us, they don't accept HIV, so I get hurt. (Female-focus group 2)

Many participants described being disowned by their families and whole communities when they discovered that they were living with HIV. Rejection by families appears to have forced some participants into homelessness, migration and situations of severe adversity that hinder treatment adherence. This experience appeared to be more common among ethnic minorities and less common in Tehran.

When I found out I didn't tell anyone, but my brother found out, collected all my clothes, and threw me out of the house. A few weeks ago, I went for my uncle's funeral. The whole family asked my brother why he made me homeless. He easily said to my parents that I have HIV, and they all disowned me. (Male-Interview 3)

I was thrown out of the house and had to leave my community when they found out I am HIV positive. I ran away and came to Tehran and for 5 years I was sleeping in the streets, under the bridges of this city. These things are more common among us [ethnic minorities]. (Male Patient-Focus group 1)

Interviewees also described acts of discrimination by medical practitioners, outside the circle of HIV experts. Nurses, dentists and general practitioners were described as having a discriminatory attitude and as refusing medical and dental care to people living with HIV. This experience was described as intensifying despair, depression and withdrawal from care.
When I go to the doctor unrelated to HIV, and they realize that I have HIV, their attitude changes. I have a glass of water and the doctor says to put the glass in the bin outside his office, or to not use the toilette in his office. Of course, this intensifies my depression. (Female patientfocus group 8)

In many cases the refusal to provide treatment to a patient occurred within the context of a breach of confidentiality within the hospital wards or clinics where they were receiving care that was unrelated to HIV.

I found out I have HIV when I was getting chemotherapy and was in my third session. The doctor told me to get tested for HIV and when I went back and told them I am positive they treated me with unbelievable avoidance. The nurses didn't even want to change my IV. They talked behind my back and told everyone in the ward that I have HIV. I just didn't go for my fourth session of chemotherapy. (Male patient-focus group 7)

An absence of dental care was described as impacting antiretroviral adherence in older patients who had not received necessary dental care and for whom food intake was compromised as a result, leading to nutritional problems that affected antiretroviral adherence.

So many dentists refused care to me, so I lose hope in them [dentists] and stop going for visits. For medication adherence, nutrition is most important, especially for older people like me. I now need to remove two of my teeth, but I can't go. (Female patient-focus group 6)

\section{Economic insecurity}

Financial insecurities are not a direct barrier to accessing treatment, given the provision of free and universal antiretroviral therapy in Iran. However, economic insecurity, caused by sanctions and inflation, appeared to have had a strong impact on the ability of people living with HIV to manage more generally, leading to feelings of despair, and in some cases suicidal thoughts. One participant described not wanting to continue with life as a result of constant price increases.

Constant price increases bring you down [emotionally], so then you have no motivation to look after yourself. Adherence to treatment in reality is looking after yourself. (Female patient-focus group 8)

This year I just can't take the prices anymore. I've been thinking about giving up on this life. I just can't stop crying. It's getting harder every day and I can't continue this life anymore. (Female patient-focus group 8)

Sometimes the cost of living is so high that you feel like you are breaking under this life, everything becomes a challenge from waking up in the morning to taking your medications. (Male patient-Interview 6)

Participants noted that they would have stopped antiretroviral therapy if it were not free, but nutritional supplements prescribed by physicians were described as becoming increasingly unaffordable. 
If they didn't give us the medicines for free I would definitely not take [antiretroviral therapy]. Because for example right now doctor told me to take vitamin $\mathrm{E}$ and to take iron because I have 'Kist' (Cystitis). I try to buy it but sometimes when I don't have enough money I don't buy. Because right now all the prices have gone up. (Female patient interview 3)

One doctor described that economic hardship drives people to profit in the black-market by selling their medication on the streets as pre-exposure prophylaxis.

We don't offer universal PrEP, so some patients who are in need go and sell their own medications as PrEP to others in the black market, so they end up not adhering properly. (Doctor-focus group with healthcare providers)

\section{Social support}

The data also suggest that the existence or lack of social support can moderate despair. Participants described how the lack of family and social support made them feel 'uncared for' and was another trigger for despair and stopping treatment.

I don't have any support. In my family there is no one to help and support me to take my medicine. I have health problems and was in the emergency room, but it wasn't even important to them. I considered stopping the medicines all together. (Female patient-interview 11)

Feeling beset by family problems unrelated to HIV, led to one participant stopping treatment because she could no longer see the value in life.

For me what causes me to not take my medicine is problems in life. I have problems with my husband, I stop taking my medicine. I face problems with my family, my father and my mother, I can't take it at points, so I really lose hope in life, and I leave my medicine aside. It's like everything loses its importance for me. Living life loses its value. (Female patient-focus group 2)

The presence of social support was also perceived to be helpful in enabling some participants to cope better with adherence.

I am very lucky because my family is very supportive. They help me a lot and even called our relatives who live in America to get information for me from mayo clinic and make sure I am getting the right care and that has been very reassuring for me. (Female patient-focus group 2)

The importance of having access to empowering classes, peer support clubs, harm reduction opportunities for quitting drugs and online support groups was highlighted by many participants. Interviewees who had taken advantage of such opportunities referred to developing a sense of self-love and an awareness of the need to take care of themselves, which they felt had protected against self-harm and non-adherence:

They teach us the art of life here. We used to even play music here, and these helped me come out of my depression. With time and counselling I realised that I am doing this [being on treatment] for myself. I have to love myself first, otherwise who will. (Male patient-interview 9)

I joined the Positive Club [Peer Support Club], they taught us a lot here. I started methadone, helped me get clean and come out of darkness and instead take care of myself. The classes taught us life-skills. I ended up even finding a job and going back into the society. (Male patient-focus group 5)

We help each other online, for example if a person runs out of medicine, we send it to them until they can go and get their pills. Our telegram groups have been helpful for us to look after each other. (Male patient-focus group 1)

Participants especially enjoyed the interaction with 'positives' (Persian term for living with HIV), and caring healthcare staff.

When I discovered the positive-club, I realised that I am not alone, and talking to other positives really gave me motivation to take care of myself. (Male patient-interview 1)

The staff here are really exceptional. Every one of them are very dear to me. Just knowing that they care gives us hope. It also helps that some of them are positives themselves and we share this pain together. (Female patient-focus group 2)

\section{DISCUSSION}

In this study, we aimed to identify pathways through which the socioecological context of living with HIV in Tehran, Iran, leads to cognitive and affective mechanisms that shape engagement with antiretroviral therapy. To the best of our knowledge, this paper presents the first in-depth exploration of engagement with and treatment adherence in a MENA country. Previous studies from MENA, most of which are from Iran, are largely composed of quantitative surveys, producing lists of risk and protective factors for treatment adherence. ${ }^{7-14}$ Our findings suggest that the three key affective and cognitive mechanisms of denial, fear and despair undermine successful and lifelong treatment. We detail the ways in which denial and fear are triggered through a context of negative societal perceptions, lack of awareness regarding HIV and widespread misinformation. While despair is described as occurring in response to experiencing HIVrelated stigma, acts of discrimination and living through economic insecurity, it is perceived to be moderated by social support. Importantly, we observed a temporal effect in terms of Iran's rapidly changing economic climate on the experience of despair and its influence on lifelong treatment.

Denial appears to be a cognitive mechanism that drives many away from treatment in an attempt to escape the negative societal perceptions regarding HIV, especially in the early stages following diagnosis. Recognised as the first of five stages of grief, ${ }^{46}$ denial was similarly identified to be a coping mechanism following HIV diagnosis. However, participants in this study retrospectively reported unusually lengthy periods of being in denial 
and delaying treatment initiation, sometimes until they experienced AIDS-related illnesses and low CD4 counts, despite the availability of treatment at the time of diagnosis. While in denial, some participants began to experiment with new classes of drugs (eg, methamphetamine) or new routes of drug administration (eg, increased injection), ultimately risking losing their life to harmful drug use.

More men than women described reacting through denial and avoidance, while more women described feelings of despair and depression. The former appears to act more as a barrier to enrolment and the latter to adherence. This potential difference between genders could be rooted in previously documented hegemonic notions of masculinity, ${ }^{48}$ whereby men view HIV as a threat to their manhood and dignity and are wary of any association with HIV. ${ }^{2649}$ In the process of denying the diagnosis, they refuse treatment and may also prevent women and other members of their household from accessing treatment services. ${ }^{26}{ }^{49}$ It was not within the scope of this study to explore how gender norms are defined within the Iranian context, or whether hegemonic notions of masculinity apply in the same way as findings from Zimbabwe in relation to HIV. ${ }^{26}{ }^{49}$ We are highly cognisant of not commenting on gender roles in stereotypical orientalising ways. Iranian society is undergoing highly rapid, dynamic and complex changes with regard to gender norms and roles. The past 40 years has expanded access to higher education, especially for women, who now make up more than half of university students, and more than sixty per cent of science and technology students. Female participation in the workforce is also rapidly increasing as influenced by an expanding service industry and by economic insecurities of single-income households. Future research can further explore if and how the changing gender norms impact the success of antiretroviral therapy in Iran and the larger MENA region.

Fear also appears to be an affective mechanism that can hinder treatment. Lifelong adherence has been more easily achieved in contexts where there is an acceptance of HIV status, ${ }^{26}{ }^{50}$ allowing patients to fit medication schedules into their lives without the impact of fear and stigma. ${ }^{51}$ Our findings suggest that in contexts were HIV has a low prevalence and high stigma, such as the MENA region, lack of societal awareness regarding HIV and misinformation regarding treatment are key contributors to fear of antiretroviral therapy. While concerns about side effects and medical anxiety are valid and that they must be addressed, study participants reported having postponed treatment initiation due to the fear associated with attending appointments or resulting from intimidating information they received regarding antiretroviral therapy. This finding corroborates previous evidence suggesting that in low HIV-prevalence settings, where the wider society still lacks awareness and knowledge about the HIV epidemic, not only does the struggle for recognition still continue, ${ }^{5}$ but daily adherence and lifelong engagement with treatment are sought in the face of much more powerful experiences of societal negative perceptions and incorrect knowledge regarding HIV. ${ }^{52}$

Despair was reported to be a key affective state in which people living with HIV reduce or intentionally stop adherence to treatment. Participants lose hope and the motivation for adherence when HIV-related stigma, enacted discrimination against HIV status and economic insecurity seem overwhelming. The experience of HIV-related stigma and discrimination has been associated with reducing treatment success in previous studies. ${ }^{23}{ }^{24}$ Here, we have identified despair as a key construct within the pathway from the social context of stigma and discrimination to treatment engagement and lifelong adherence. This finding adds to a more recent body of evidence, highlighting despair as a new driver of morbidity and mortality. ${ }^{53}$

Importantly, despair appears to be manifested when economic stagnation dominates the lives of individuals. ${ }^{54} 55$ The phenomenon of 'deaths of despair' was first identified in the USA, suggesting that social and economic immobility produce morbidity and mortality through despair, demanding public health attention. ${ }^{54} 55$ Following the 2018 reimposition of sanctions on Iran, the Iranian Rial lost half of its value during this study's data collection period, ${ }^{56}$ with continued devaluation and steep inflation rates to the present time. ${ }^{57}$ Many participants expressed loss of hope and suicidal thoughts in response to the continuously increasing economic pressures they felt and their decreasing ability to manage financially. This finding suggests new causes of morbidity and mortality linked to despair that is driven in a climate of economic insecurity, sanctions and inflation. It appears that for Iranians living with HIV, despair caused by economic insecurity can undermine motivation for adherence, drive intentional disengagement with care and lead to suicidal thoughts. While global health studies often ignore the health impacts of different economic realities, the findings of the current study suggest the need for further investigation into despair-driven adverse health events within the context of such realities, particularly for vulnerable groups such as people living with HIV.

Moreover, this study sheds light on the importance of people's behaviours and responses within the context of their lives as lived through time. ${ }^{58}$ For instance, in Iran, the drive to harmful drug use must be understood within a context in which people lose hope, or rather stay in denial, in the face of HIV-related stigma, discrimination and misinformation, sometimes resulting from low awareness at a societal level. The HIV epidemic is highly dynamic in terms of transmission routes, the populations affected and the resources available at any given social and economic time point. ${ }^{5960}$ Drug use patterns in Iran have changed substantially over time,${ }^{60}$ from an older generation that smoked opium to a younger generation that injects heroin, and a recent increase in methamphetamine use across all ages and socioeconomic groups. ${ }^{60}$ 
These transitions have been linked to drastic political and economic changes, from the aftermath of an 8-year war to over three decades of sanctions, inflation and un(der)employment, ${ }^{59}$ thereby revealing the impact of temporal junctures in which politics and economics can have a significant direct and indirect impact in terms of health outcomes.

Our findings also show that in the face of adversities linked to living with HIV, social support plays a key role in enhancing or reducing hope. Perceived lack of support, especially in terms of unsupportive family members, appears to fuel despair and disrupt adherence, with some participants reporting intentionally stopping adherence as a result of not feeling cared for. Moreover, competent healthcare providers and peers can have a positive impact in helping people living with HIV overcome difficulties in making sense of their experiences and negotiating support. Many antiretroviral users indicated the rewarding and positive relationships with healthcare staff and peer supporters that were viewed as enabling them to gain optimal benefits from the healthcare system. Access to caring support appears to dilute the experiences of stigma and discrimination from the wider society. Participants especially enjoyed the interaction with peers living with HIV, through which a feeling of 'us'-the 'positives' in Persian - and the uninformed and hostile 'others' enables an empowering distinction. Online and offline support networks in which patients give and receive supportive information appear to be particularly valued.

At its onset, the HIV epidemic in Iran was producing more deaths by suicide than HIV-related causes. ${ }^{5961}$ In response, community outreach programmes mobilised to provide culturally sensitive support and counselling to HIV-affected families who had disowned their kin who were diagnosed with HIV. ${ }^{6263}$ This work was followed by the development of triangular clinics, which provided treatment for drug use, HIV and other sexually transmitted diseases; a model that diffused stigma by avoiding direct reference to HIV/AIDS and resulting in a growing uptake of HIV testing. ${ }^{61}$ Other national strategies to support those living with and those at risk of HIV include peer support clubs, harm reduction programmes, methadone maintenance treatment, needle exchange programmes and free counselling for people who use drugs. ${ }^{63}$ These programmes have reduced the number of people living with HIV who turn to suicide and have improved harm reduction for people who use drugs. ${ }^{59}$ Yet, given that the HIV epidemic in Iran remains hidden from the general public and is absent from public debate, universal access to antiretroviral therapy is failing to achieve its promise in the absence of public recognition and support for people living with HIV. Therefore, more interventions that are culturally appropriate, contextually sensitive and tailored to the dynamically changing needs of individuals are necessary to improve the lived experience of Iranians living with HIV. ${ }^{58}$

The findings from this study have informed an intervention for improving engagement with and adherence to antiretroviral therapy for people living with HIV in Iran. Rather than simply orienting the intervention to the outcome of improving adherence, the findings from this study have been used to develop a personalised mobile health intervention that is oriented to Iran's particular socioecological context, tailored to individual cognitive and affective states, and attuned to the political and economic climate of the time.$^{58}$ Previous evidence demonstrates that mobile health strategies are a low-resource option that can make people living with HIV feel cared for, ${ }^{64}$ offer tailored and temporally adaptive support and maintain patient privacy. ${ }^{65-67}$ Such digital strategies have the added benefit of augmenting existing infrastructure (eg, harm-reduction and peer support programmes) to enable patients in making optimal use of available services. ${ }^{68}{ }^{69}$ While public awareness campaigns are chiefly needed to change the attitude of Iranian society towards HIV, personalised mobile health strategies will help people living with HIV to benefit more widely from available care and mitigate the experiences of 'deadly stigma'."

\section{Strengths and limitations}

This qualitative study provided participants with an opportunity to discuss their perceptions of Iran's HIV context and define their responses to that context, thereby giving voice to a population living within the hidden context of the HIV epidemic in Iran. This enabled us to capture the nuances of experience in terms of living within HIV within this particular socioecological context and thereby to identify the cognitive and affective states that can shape antiretroviral use. Moreover, the coding and analysis was conducted in Persian by two independent researchers, who are native speakers of the language. This approach ensured that the essence of language, used by participants, was not lost within the analysis of the emerging themes.

Participation in this study was voluntary with recruitment from an HIV/AIDS clinic, and the sample may have been biased toward more motivated patients, who were able to overcome and report retrospectively on multiple barriers to lifelong treatment, including (but not limited to) stigma, transportation costs and opportunity costs. However, in accordance with principles of social constructivist research ${ }^{70}$ rather than seeking to generate characterisations of all members of a statistically representative sample, an in-depth exploration of a small sample was undertaken to identify the diverse ways that the lived experience of people living with HIV influences their reasoning and reactions in terms of their relationship with antiretroviral therapy.

\section{CONCLUSIONS}

Our findings suggest that engagement with and adherence to antiretroviral therapy are disrupted when people living with HIV respond to Iran's socioecological context through the cognitive and affective states of denial, fear and despair. Therefore, in addition to the availability of free antiretroviral therapy, more awareness about HIV is needed to prevent 
the rejection of treatment caused by denial and fear, and to provide hope and motivation for lifelong engagement with antiretroviral therapy. We observe that the low prevalence of HIV in Iran has stymied societal awareness and knowledge regarding HIV and antiretroviral therapy. Moreover, we note that the profound experiences of despair, in response to harsh economic conditions, jeopardise hope and motivation for continued adherence. Our findings support recent calls for the study of global health not be decoupled from that of international power politics, ${ }^{71}$ and emphasises the importance of understanding the nuances of experience that impact health decisions within the social and structural context of HIV. ${ }^{36} 3872$

Twitter Vira Ameli @viraameli

Acknowledgements We acknowledge the members of the Iranian Research Centre for HIV/AIDS (IRCHA): Baran Amiri and Seyed Ahmad Seyed Alinaghi, the Voluntarily, Counselling and Testing Centre at Imam Khomeini Hospital: Abbas Boosiraz and Mitra Sheikhan and the Positive Club at Imam Khomeini Hospital: Zahra Bayat Jozani and Nasrin Kordi Ardakani, for their invaluable contributions in informing and facilitating this study.

Contributors VA is the guarantor and responsible for the overal content, conceived the study, collected data, drafted and finalised the manuscript. VA and MM are the grant holders of this study. VA and LT transcribed, coded and analysed the data. JH, LS, FM and JB provided critical input to finalise the paper. Finally, LT, JB, LS, FM, JH and MM contributed to this study and this manuscript by critically commenting and approving the final manuscript.

Funding This study is funded as part of a grant from Tehran University of Medical Sciences (97-02-55-38693), Ministry of Health and Medical Education, Tehran, Iran.

Disclaimer The funder does not have any role in the design of the study. The views expressed are those of the authors and not necessarily those of the funder.

Competing interests None declared.

Patient consent for publication Not applicable.

Ethics approval Ethical approval was granted by the Tehran University of Medical Sciences and Oxford Tropical Research Ethics Committee.

Provenance and peer review Not commissioned; externally peer reviewed.

Data availability statement Data are available on reasonable request. Transcripts from interviews and focus group discussions are available upon reasonable request from the corresponding author. All transcripts are saved in the original language of respondents.

Supplemental material This content has been supplied by the author(s). It has not been vetted by BMJ Publishing Group Limited (BMJ) and may not have been peer-reviewed. Any opinions or recommendations discussed are solely those of the author(s) and are not endorsed by BMJ. BMJ disclaims all liability and responsibility arising from any reliance placed on the content. Where the content includes any translated material, BMJ does not warrant the accuracy and reliability of the translations (including but not limited to local regulations, clinical guidelines, terminology, drug names and drug dosages), and is not responsible for any error and/or omissions arising from translation and adaptation or otherwise.

Open access This is an open access article distributed in accordance with the Creative Commons Attribution Non Commercial (CC BY-NC 4.0) license, which permits others to distribute, remix, adapt, build upon this work non-commercially, and license their derivative works on different terms, provided the original work is properly cited, appropriate credit is given, any changes made indicated, and the use is non-commercial. See: http://creativecommons.org/licenses/by-nc/4.0/.

\section{REFERENCES}

1 Abu-Raddad LJ, Akala FA, Semini I, et al. Characterizing the HIVI AIDS epidemic in the middle East and North Africa, 2010. https://doi. org/10.1596/978-0-8213-8137-3

2 UNAIDS. AIDS info, 2021. Available: https://aidsinfo.unaids.org/
3 Alabaster O. Deadly stigma: HIV Infections in Middle East on the Rise - VICE. Available: https://www.vice.com/en_asia/article/xy84jn/ hiv-infections-are-growing-in-the-middle-east-thanks-to-prejudiceand-a-lack-of-sex-ed

4 Gökengin D, Doroudi F, Tohme J, et al. HIV/AIDS: trends in the Middle East and North Africa region. Int J Infect Dis 2016;44:66-73.

5 Dejong J, Mortagy I. The struggle for recognition by people living with HIV/AIDS in Sudan. Qual Health Res 2013;23:782-94.

6 Kamarulzaman A. Fighting the HIV epidemic in the Islamic world. Lancet 2013;381:2058-60.

7 Moayed MS, Khatiban M, Nassiri Toosi M, et al. Barriers to adherence to medical care programs in liver transplant recipients: a qualitative study. Int J Organ Transplant Med 2019;10:115-26.

8 Mohraz M, Tayeri K, Namdari Tabar H, et al. Evaluation of acquired HIV drug resistance among people living with HIV who have taken antiretroviral therapy for 9-15 months in 14 triangular clinics in Iran, 2015-2016. Intervirology 2018;61:292-300.

9 Farrokhi M, Gholami M, Mohraz M, et al. HIV drug resistance among naïve HIV-infected patients in Iran. J Res Med Sci 2019;24:31.

10 Sadeghi L, Lolaie M, Tabatabai RA, et al. HIV-1 drug resistance profiles for the HIV protease and reverse transcriptase gene in patients receiving combination therapy in Tehran, Iran. Infect Disord Drug Targets 2018;18:241-8.

11 Motazedian N, Sayadi M, Firoozbakhtian A. Non-adherence to antiretroviral medication in Shiraz, 2014: a cross sectional study. Afr Health Sci 2018;18:384-93.

12 Khalili H, Rohani R, Seyedalinaghi S, et al. Adherence to antiretroviral therapy among Iranian HIV/AIDS patients. Curr Clin Pharmacol 2012;7:111-5.

13 Morowatisharifabad M-A, Movahed E, Nikooie R, et al. Adherence to medication and physical activity among people living with HIV/AIDS. Iran J Nurs Midwifery Res 2019;24:397-9.

14 Seyed Alinaghi SA, Farhoudi B, Mohraz M, et al. Adherence to antiretroviral therapy and tuberculosis treatment in a prison of Tehran, Iran. Infect Disord Drug Targets 2016;16:199-203.

15 Arrivillaga M, Ross M, Useche B, et al. Applying an expanded social determinant approach to the concept of adherence to treatment: the case of Colombian women living with HIV/AIDS. Womens Health Issues 2011;21:177-83.

16 Gourlay A, Birdthistle I, Mburu G, et al. Barriers and facilitating factors to the uptake of antiretroviral drugs for prevention of motherto-child transmission of HIV in sub-Saharan Africa: a systematic review. J Int AIDS Soc 2013;16:18588.

17 Kaufman MR, Cornish F, Zimmerman RS, et al. Health behavior change models for HIV prevention and AIDS care: practical recommendations for a multi-level approach. J Acquir Immune Defic Syndr 2014;66:S250-8.

18 Castro EM, Santiago LE, Jiménez JC, et al. A social-ecological view of barriers and facilitators for HIV treatment adherence: interviews with puerto rican HIV patients. PLoS One 2015;10:e0125582.

19 Yakob B, Ncama BP. A socio-ecological perspective of access to and acceptability of HIV/AIDS treatment and care services: a qualitative case study research. BMC Public Health 2016;16:155.

20 Mukumbang FC, Mwale JC, van Wyk B. Conceptualising the factors affecting retention in care of patients on antiretroviral treatment in Kabwe district, Zambia, using the ecological framework. AIDS Res Treat 2017;2017:7356362.

21 Berkman A, Garcia J, Muñoz-Laboy M, et al. A critical analysis of the Brazilian response to HIV/AIDS: lessons learned for controlling and mitigating the epidemic in developing countries. Am J Public Health 2005;95:1162-72.

22 Masanjala W. The poverty-HIV/AIDS nexus in Africa: a livelihood approach. Soc Sci Med 2007;64:1032-41.

23 Tsai AC, Bangsberg DR, Kegeles SM, et al. Internalized stigma, social distance, and disclosure of HIV seropositivity in rural Uganda. Ann Behav Med 2013;46:285-94.

24 Katz IT, Ryu AE, Onuegbu AG, et al. Impact of HIV-related stigma on treatment adherence: systematic review and meta-synthesis. $J$ Int AIDS Soc 2013;16:18640

25 Cluver L, Meinck F, Toska E, et al. Multitype violence exposures and adolescent antiretroviral nonadherence in South Africa. AIDS 2018;32:975-83.

26 Skovdal M, Campbell C, Nyamukapa C, et al. When masculinity interferes with women's treatment of HIV infection: a qualitative study about adherence to antiretroviral therapy in Zimbabwe. J Int AIDS Soc 2011:14:29

27 Govindasamy D, Ford N, Kranzer K. Risk factors, barriers and facilitators for linkage to antiretroviral therapy care: a systematic review. AIDS 2012;26:2059-67.

28 Cluver L, Pantelic M, Toska E, et al. STACKing the odds for adolescent survival: health service factors associated with full 
retention in care and adherence amongst adolescents living with HIV in South Africa. J Int AIDS Soc 2018;21:e25176.

29 Pecoraro A, Royer-Malvestuto C, Rosenwasser B, et al. Factors contributing to dropping out from and returning to HIV treatment in an inner city primary care HIV clinic in the United States. AIDS Care 2013;25:1399-406.

30 Rhodes T, Cusick L. Love and intimacy in relationship risk management: HIV positive people and their sexual partners. Sociol Health IIIn 2000;22:1-26.

31 Friedland J, Renwick R, Mccoll M. AIDS care: psychological and socio-medical aspects of AIDS / HIV coping and social support as determinants of quality of life in HIV/AIDS C oping and social support as determ inants of quality of life in H IV/AIDS. AIDS Care Psychol Socio-Medical Asp 2010;8:37-41.

32 Mills JC, Pence BW, Edmonds A, et al. The impact of cumulative depression along the HIV care continuum in women living with HIV during the era of universal antiretroviral treatment. J Acquir Immune Defic Syndr 2019;82:225-233.

33 Bar-Lev S. 'We are here to give you emotional support': performing emotions in an online HIV/AIDS support group 2008.

34 Westergaard RP, Hess T, Astemborski J, et al. Longitudinal changes in engagement in care and viral suppression for HIV-infected injection drug users. AIDS 2013;27:2559-66.

35 Leylabadlo HE, Baghi HB, Fallahi L, et al. From sharing needles to unprotected sex: a new wave of HIV infections in Iran? Lancet HIV 2016;3:e461-2.

36 Campbell C, Cornish F. Towards a 'fourth generation' of approaches to HIV/AIDS management: creating contexts for effective community mobilisation. AIDS Care 2010;22:1569-79.

37 Seeley J, Watts $\mathrm{CH}$, Kippax S, et al. Addressing the structural drivers of HIV: a luxury or necessity for programmes? J Int AIDS Soc 2012;15:1-4

38 Gupta GR, Parkhurst JO, Ogden JA, et al. Structural approaches to HIV prevention. Lancet 2008;372:764-75.

39 Dalkin SM, Greenhalgh J, Jones D, et al. What's in a mechanism? Development of a key concept in realist evaluation. Implement Sci 2015;10:49.

40 Pope C, Ziebland S, Mays N. Qualitative research in health care. analysing qualitative data. BMJ 2000;320:114-6.

41 Mir R, Watson A. Critical realism and constructivism in strategy research: toward a synthesis. Strateg Manag J 2001;22:1169-73.

42 Bogna F, Raineri A, Dell G. Critical realism and constructivism: merging research paradigms for a deeper qualitative study. QROM 2020;15:461-84.

43 Denzin NK, Lincoln Y. Contents and index. In: Handbook of qualitative research, 2011.

44 Palinkas LA, Horwitz SM, Green CA, et al. Purposeful sampling for qualitative data collection and analysis in mixed method implementation research. Adm Policy Ment Health 2015;42:533-44.

45 Attride-Stirling J. Thematic networks: an analytic tool for qualitative research. Qual Res 2001;1:385-405.

46 The HIV 5 stages of grief and how to cope with them. Available: http://www.ias-2005.org/support/hiv-5-stages-of-grief-how-to-cope

47 Connell R. Masculinities, 2005.

48 Courtenay WH. Constructions of masculinity and their influence on men's well-being: a theory of gender and health. Soc Sci Med 2000;50:1385-401.

49 Skovdal M, Campbell C, Madanhire C, et al. Masculinity as a barrier to men's use of HIV services in Zimbabwe. Global Health 2011;7:13

50 Campbell C, Skovdal M, Madanhire C, et al. "We, the AIDS people...": how antiretroviral therapy enables Zimbabweans living with HIV/AIDS to cope with stigma. Am J Public Health 2011;101:1004-10.

51 Dahab M, Charalambous S, Hamilton R, et al. "That is why I stopped the ART": patients' \& providers' perspectives on barriers to and enablers of HIV treatment adherence in a South African workplace programme. BMC Public Health 2008;8:63.

52 Genberg BL, Hlavka Z, Konda KA, et al. A comparison of HIV/AIDSrelated stigma in four countries: negative attitudes and perceived acts of discrimination towards people living with HIV/AIDS. Soc Sci Med 2009;68:2279-87.

53 Shanahan L, Hill SN, Gaydosh LM, et al. Does despair really kill? A roadmap for an evidence-based answer. Am J Public Health 2019;109:854-8.

54 Case A, Deaton A. Rising morbidity and mortality in midlife among white non-Hispanic Americans in the 21st century. Proc Natl Acad Sci U S A 2015;112:15078-83.

55 Case A, Deaton A. Deaths of despair and the future of capitalism. Princeton University Press, 2020.

56 Al Jazeera. Iran's currency plunges to record low as US sanctions loom, Business and Economy News. Available: https://www. aljazeera.com/economy/2018/7/29/irans-currency-plunges-torecord-low-as-us-sanctions-loom

57 Reuters. Iranian rial drops to lowest rate since September 2018 against the U.S. dollar. Available: https://www.reuters.com/article/ iran-currency-idUSL8N2CU7G1

58 Beckmann N. Medicines of hope? The tough decision for antiretroviral use for HIV in Zanzibar, Tanzania. J East African Stud 2012;6:690-708.

59 Behrouzan O. An epidemic of meanings: the significance of history, gender and language in HIV/AIDS epidemics in the fourth wave: Violence, Gender, Culture and HIV in the 21st Century 2010.

60 Ghiabi M. Drugs politics: managing disorder in the Islamic Republic of Iran 2019.

61 Rosenberg J, Bohrer M, Rhatigan J. Iran's Triangular Clinic. Cases in Global Health Delivery 2011.

62 The New York Times. How Iran derailed a health crisis. Available: https://opinionator.blogs.nytimes.com/2010/12/03/how-iranderailed-a-health-crisis/

63 Rahnama R, Mohraz M, Mirzazadeh A, et al. Access to harm reduction programs among persons who inject drugs: findings from a respondent-driven sampling survey in Tehran, Iran. Int $J$ Drug Policy 2014;25:717-23.

64 Ware NC, Pisarski EE, Tam M, et al. The meanings in the messages: how SMS reminders and real-time adherence monitoring improve antiretroviral therapy adherence in rural Uganda. AIDS 2016;30:1287-94.

65 Chiasson MA, Hirshfield S, Rietmeijer C. HIV prevention and care in the digital age. J Acquir Immune Defic Syndr 2010;55:S94-7.

66 Noar SM, Black HG, Pierce LB. Efficacy of computer technologybased HIV prevention interventions: a meta-analysis. AIDS 2009;23:107-15.

67 Noar SM. Computer technology-based interventions in HIV prevention: state of the evidence and future directions for research. AIDS Care 2011;23:525-33.

68 WHO. Recommendations on digital interventions for health system strengthening, 2019. Available: https://apps.who.int/iris/bitstream/ha ndle/10665/311941/9789241550505-eng.pdf?ua=1

69 Spies G, Asmal L, Seedat S. Cognitive-behavioural interventions for mood and anxiety disorders in HIV: a systematic review. J Affect Disord 2013;150:171-80.

70 Flyvbjerg B. Making social science matter: why social inquiry fails and how it can succeed again. Vol. 7. Cambridge University Press, 2001.

71 Benatar S. Politics, power, poverty and global health: systems and frames. Int J Health Policy Manag 2016;5:599-604.

72 Castro A, Farmer P. Understanding and addressing AIDS-related stigma: from anthropological theory to clinical practice in Haiti. Am J Public Health 2005;95:53-9. 